\title{
Cancer-associated fibroblast suppresses killing activity of natural killer cells through downregulation of poliovirus receptor (PVR/CD155), a ligand of activating NK receptor
}

\author{
TOMOKO INOUE, KATSUYUKI ADACHI, KEI KAWANA, AYUMI TAGUCHI, TAKESHI NAGAMATSU, \\ ASAHA FUJIMOTO, KENSUKE TOMIO, AKI YAMASHITA, SATOKO EGUCHI, HARUKA NISHIDA, \\ HIROE NAKAMURA, MASAKAZU SATO, MITSUYO YOSHIDA, TAKAHIDE ARIMOTO, \\ OSAMU WADA-HIRAIKE, KATSUTOSHI ODA, YUTAKA OSUGA and TOMOYUKI FUJII
}

Department of Obstetrics and Gynecology, Faculty of Medicine, The University of Tokyo, Bunkyo-ku, Tokyo 113-8655, Japan

Received April 26, 2016; Accepted July 14, 2016

DOI: 10.3892/ijo.2016.3631

\begin{abstract}
Cancer-associated fibroblasts (CAFs) play an important role in cancer expansion and progression in tumor microenvironment (TME), via both direct and indirect interactions. Natural killer $(\mathrm{NK})$ cells play a crucial role in anticancer immunity. We investigated the inhibitory effects of CAFs on NK cell activity. CAFs were isolated from endometrial cancer tissue, while normal endometrial fibroblasts (NEFs) were obtained from normal endometrium with no pathological abnormality. NK cells were obtained from allogenic healthy volunteers. CAFs or NEFs were co-cultured at an $\mathrm{NK} /$ fibroblast ratio of 1:1 with or without inserted membrane. For NK cell activity, K562 cells were cultured as target cells. NK cell-killing activity was determined by calculating the ratio of PI-positive K562 cells in the presence of NK cells co-cultured with fibroblasts versus NK cells alone. To examine whether NK cell activity was suppressed by IDO pathway, we inhibited IDO activity using the IDO inhibitor 1-MT. We demonstrated that CAFs derived from endometrial cancer induced greater suppression of the killing activity of allogenic NK cells compared with normal endometrial fibroblasts (NEFs). The suppression of NK cell activity by CAFs was inhibited when a membrane was inserted between the CAFs and NK cells, but not by 1-MT, an inhibitor of IDO.
\end{abstract}

Correspondence to: Dr Kei Kawana or Dr Katsuyuki Adachi, Department of Obstetrics and Gynecology, Graduate School of Medicine, The University of Tokyo, 7-3-1 Hongo, Bunkyo-ku, Tokyo 113-8655, Japan

E-mail: kkawana-tky@umin.org

E-mail: kadachi-gyn@umin.org

Abbreviations: CAFs, cancer-associated fibroblasts; TME, tumor microenvironment; PVR, poliovirus receptor; NEFs, normal endometrial fibroblasts; IDO, indoleamine 2,3-dioxygenase

Key words: cancer-associated fibroblast, endometrial cancer, natural killer cell, killing activity, poliovirus receptor (PVR/CD155)
We focused on receptor-ligand interactions between CAFs and $\mathrm{NK}$ cell and found that cell-surface poliovirus receptor (PVR/ CD155), a ligand of activating NK receptor DNAM-1, was downregulated in the CAFs compared with NEFs. To confirm whether PVR downregulation results in the decrease of NK cell-killing activity, PVR expression in NEFs was knocked down using siRNA against PVR (PVRsi). NK cell activity was suppressed by co-culture with PVR-knockdown NEFs, to a similar extent than CAF-induced suppression. CAFs showed increased suppression of NK cell-killing activity compared with NEFs, due to decreased PVR cell surface expression, a ligand of an NK activating receptor. This study demonstrated a novel mechanism of suppression of NK cell activity by CAFs in the TME.

\section{Introduction}

Cancer-associated fibroblasts (CAFs) regulate not only carcinogenesis, but also the immune evasion of cancer in the tumor microenvironment (TME), which facilitates cancer cell proliferation, expansion, and metastasis (1-5). The TME is comprised of various types of cells, including microvascular endothelial cells, immune cells, and CAFs, and these cells interact directly and indirectly with each other maintaining homeostasis of the TME. Among these cells, CAFs are important regulators of the immunological microenvironment of the tumor. Sanz-Moreno et al reported that CAFs regulate immune evasion in the TME by various mechanisms, including the use of cytokines and cell attachment (6). They demonstrated that the ROCK and JAK1 signaling pathway generates a contractile force in stromal fibroblasts, allowing remodeling of the extracellular matrix and the creation of tracks for the collective migration of squamous carcinoma cells. Furthermore, Gaggioli et al demonstrated that the generation of these tracks by fibroblasts was sufficient in enabling collective invasion of squamous cell carcinoma cells (7).

NK cells play an important role in cancer immunity in the TME. A review by Chan et al identified several well-known ligands of NK paired or activating receptors that are expressed on the cell surface of target cells, including malignant cells 
(8). NK activating receptors include NKp30, NKp44, NKp46, NKG2D, DNAX accessory molecule-1 (DNAM-1), and LFA-1 (9). In addition, indoleamine 2,3-dioxygenase (IDO) is produced by various malignant cells, inactivates NK cells, and evades the immune system in the TME (10).

Poliovirus receptor (PVR/CD155) is a ligand of the paired NK receptors, DNAM-1 (activating) and TIGIT (inhibiting). NK cells can kill cancer cells expressing PVR via the DNAM1-mediated activating signaling $(11,12)$. Several studies have demonstrated that PVR overexpression in cancer cells significantly affects their migration, invasion, proliferation, and metastasis (13). Although these previous studies have investigated the interactions between NK cells and malignant cells, there are few reports investigating the interaction of CAFs with NK cells. A previous study reported that CAFs inhibit the IL-2-induced cell-surface expression of the activating NK receptors, NKp44, NKp30, and DNAM-1 (9). However, there have been no studies investigating the association between NK cell activity and PVR expression in CAFs.

Considering the NK cell-mediated immune evasion mechanisms in the TME, we hypothesized that in addition to malignant cells, CAFs may also play a role in the suppression of NK cell activity in the TME. In this study, we used CAFs and normal endometrial fibroblasts (NEFs), derived from endometrial cancer and normal endometrial stroma, respectively. In the uterine endometrium, endometrial stroma is enriched in fibroblasts and surrounds the endometrial glandular epithelia, and these NEFs can be transformed to CAFs in endometrial cancer. Therefore, the use of endometrial cancer is suitable for comparison between CAFs and NEFs. In this study, we investigated the inhibitory effect of CAFs on NK cell-killing activity and the underlying mechanism.

\section{Materials and methods}

Patients and establishment of fibroblasts. Tumor samples were obtained from the patients with endometrial carcinoma, and normal endometrium were collected from those without pathology in uterine endometrium, undergoing surgical resection in our hospital. All women gave written informed consent and the Research Ethics Committee of the University of Tokyo approved all aspects of the study.

CAFs were isolated from the cancer tissues of endometrial cancer while NEFs were from the normal endometrium with no pathological abnormality. The tissues were minced and digested in DMEM/F12 medium, (Gibco, Japan), supplemented with $100 \mathrm{IU} / \mathrm{ml}$ penicillin, $100 \mu \mathrm{g} / \mathrm{ml}$ streptomycin, $1 \mathrm{mg} / \mathrm{ml}$ collagenase type I (Wako, Tokyo, Japan), and $25 \mathrm{ng} / \mathrm{ml}$ DNase, Roche Diagnostics $\mathrm{GmbH}$ at $37^{\circ} \mathrm{C}$ for $60 \mathrm{~min}$, filtered with 100 and $70 \mu \mathrm{m}$ cell strainers, BD Falcon, and centrifuged at 1,500 rpm for $5 \mathrm{~min}$ and washed with D-PBS, Wako. They were resuspended in DMEM/F12 with 10\% FBS, $100 \mathrm{IU} / \mathrm{ml}$ penicillin, $100 \mu \mathrm{g} / \mathrm{ml}$ streptomycin, and cultured at $37^{\circ} \mathrm{C}$ in humidified $5 \% \mathrm{CO}_{2}$ environment. Fibroblasts passaged for 2-8 passages were used before the experiments.

Isolation of NK cells and NK cell/fibroblast co-cultures. NK cells were obtained from healthy volunteers after Ficoll-Paque gradient and negative magnetic selection, using human NK cell isolation kit from Miltenyi Biotec. NK cell purity was $>95 \%$ as evaluated by flow cytometry. They were cultured in RPMI with $10 \%$ FBS, $100 \mathrm{IU} / \mathrm{ml}$ penicillin, $100 \mu \mathrm{g} / \mathrm{ml}$ streptomycin, at $37^{\circ} \mathrm{C}$ in humidified $5 \% \mathrm{CO}_{2}$ atmosphere, and stimulated

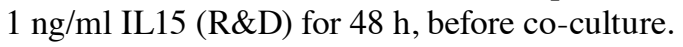

For co-culture experiments, CAFs or NEFs were seeded at $5 \times 10^{4}$ in 24-well plates with $500 \mu \mathrm{l}$ of medium and cultured for $48 \mathrm{~h}$. NK cells were added at $1 \times 10^{5}$ well, at an NK/fibroblasts ratio of $1: 1$, with or without $1-\mu \mathrm{m}$ pore of cell-culture-insert. NK cells alone were also cultured in the absence of fibroblasts. After 24-h incubation, NK cells were harvested and analyzed. We confirmed that allogenic NK cells did not kill these fibroblasts during co-culture (data not shown).

NK cell killing assay. K562 cells (obtained from ATCC; American Type Culture Collection, VA, USA) were cultured in RPMI-1640 supplemented with $10 \%$ FBS containing $100 \mathrm{U} / \mathrm{ml}$ penicillin and $100 \mu \mathrm{g} / \mathrm{ml}$ streptomycin. K562 cells were resuspended at $1-2 \times 10^{7}$ cells $/ \mathrm{ml}$ in $0.1 \%$ FBS/PBS and added CFSE, using CFSE Cell Division Assay kit from Cayman chemical, to a final concentration of $2.5 \mu \mathrm{M}$ CFSE staining solution, incubated cells at $37^{\circ} \mathrm{C}$ for $30 \mathrm{~min}$. After centrifugation of cells at $300 \mathrm{~g}$ for $10 \mathrm{~min}$, supernatant was discarded and they were resuspended into RPMI medium and incubated at $37^{\circ} \mathrm{C}$ for $30 \mathrm{~min}$. The cells were washed with $2 \% \mathrm{FBS} / \mathrm{PBS}$ three times, and $2 \times 10^{4}$ of stained $\mathrm{K} 562$ cells in $200 \mu 1$ medium were added to tubes. Harvested NK cells were diluted at $1 \times 10^{6}$ cells $/ \mathrm{ml}$ and prepare dilution series $2 \times 10^{5}$ cells per $200 \mu \mathrm{l}$. After incubation the tubes at $37^{\circ} \mathrm{C}$ for $4 \mathrm{~h}, 5 \mu \mathrm{l}$ of PI (propidium iodide solution, Biolegend, San Diego, CA, USA) was added for dead cell count. Percentage of PI-positive dead K562 cells (\% K562 PI-positive cell) was evaluated by flow cytometry. In some experiments, allogenic NK cells were incubated for $24 \mathrm{~h}$ with NEFs, CAFs or no fibroblasts followed by exposure to target (K562) cells. NK cell killing activities were indicated by ratio of $\%$ K562 PI positive cells under each condition against that of NK cells only.

Immunocytochemistry. CAFs were grown on coverslips, then serum deprived and fixed in 4\% paraformaldehyde, permeabilized with $0.1 \%$ Triton X-100, washed twice with PBS, and incubated for $60 \mathrm{~min}$ at room temperature with an anti- $\alpha$-SMA antibody (mouse clone 1A4, ab7817, Abcam MA, USA), labeled with Alexa Flour 488 (Zenon). After incubation, the slides were washed with PBS and fixed with $4 \%$ paraformaldehyde. The cells were counterstained with 4',6-diamidino-2-phenylindole dihydrochloride (DAPI).

Western blotting. CAFs and NEFs grown in chamber slides were fixed and each of them were collected and total proteins were extracted. Immunoblotted with anti-beta Actin antibody (rabbit polyclonal antibody, Abcam), and $\alpha$-SMA (mouse clone 1A4, ab7817, Abcam).

Flow cytometry. Cultured cells were harvested and incubated with PerCP Cy5.5-conjugated CD90 (mouse clone Thy1, BioLegend), FITC-conjugated Vimentin (mouse clone RV202, ab8978, Abcam), PE-conjugated PVR (mouse clone SKII.4, BioLegend) or appropriate isotype control at $4^{\circ} \mathrm{C}$ for $30 \mathrm{~min}$, washed twice, and analyzed using a BD FACS Calibur cytometer. The results were analyzed using Kaluza software. 

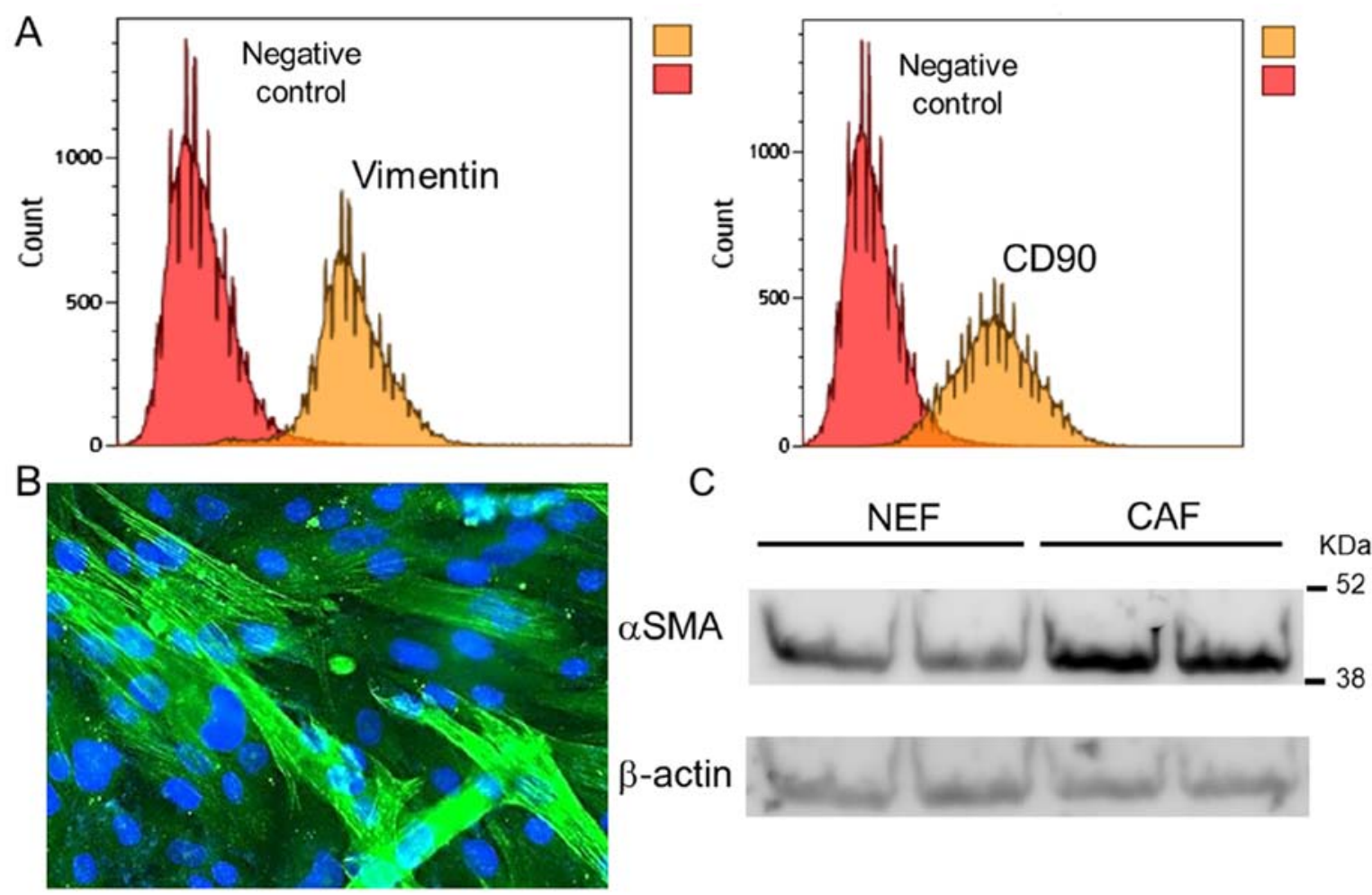

Figure 1. Difference of marker expression between NEFs and CAFs. Fibroblasts were collected from endometrial cancer or normal endometrial tissues. (A) Flow cytometry revealed CAFs expressed Vimentin and CD90 which were major marker of fibroblast. (B) Immunocytochemistry showed that CAFs expressed $\alpha$-SMA, well-known as CAF makers (green, $\alpha$-SMA; blue, DAPI). (C) Expression level of $\alpha$-SMA in CAFs was assessed by western blotting comparing with NEFs.

siRNA experiments. NEFs were transfected with 100 pmol of siRNA with Lipofectamine RNAiMAX. The complexes of RNAi and $1.5 \mu 1$ Lipofectamine RNAiMAX in $140 \mu 1$ Opti-MEM I medium without serum. Then the complexes were added in the cultured cells at $4 \times 10^{4}$ in 24 -well plates, and $500 \mu 1$ of complete medium without antibiotics was gently added in each well, and incubated for $12 \mathrm{~h}$ at $37^{\circ} \mathrm{C}$ in a $\mathrm{CO}_{2}$ incubator to be ready for the next assay of gene knockdown. To knock down PVR, siRNA by R\&D was used. The sequences were as follows: human PVR; sense, 5'-rCrArGrCUrAUUr CrGrG rArCUrCrCrArArATT; antisense, 5'-UUArGrGrArCUr CrCrGrArAUrArGrCUrGTT. The negative siRNA controls were obtained from Life Technologies.

Statistical analysis. Data are presented as means \pm SEM. Statistical analyses were carried out using Student's t-test or Dunnett analysis using JMP software. A value of $p<0.05$ was considered significant. Asterisks indicate those comparisons with statistical significance $(\mathrm{p}<0.05)$.

\section{Results}

Isolation of CAFs from endometrial cancer. To investigate the difference in effect on NK cell activity between NEFs and CAFs, we isolated fibroblasts derived from normal endometrium (NEFs) and endometrial cancer tissue (CAFs). Uterine endometrium is composed of many glands and abundant stroma. The endometrial stroma is enriched in fibroblasts, and surrounds the endometrial glandular epithelium. Endometrial cancer can transform normal fibroblasts in the stroma into CAFs. Therefore, the comparison between endometrial CAFs and NEFs was thought to be suitable for the investigation of CAF activity. Fibroblasts were isolated from cancer tissue or normal endometrium by standard isolation methods and identified by immunostaining and western blotting. Fibroblasts isolated from endometrial cancer expressed the fibroblastic markers vimentin and CD90 (Fig. 1A). To distinguish the cancer-derived fibroblasts from the NEFs, presence of $\alpha$-SMA was assessed by immunocytochemistry (Fig. 1B), and expression level of $\alpha$-SMA compared with NEFs was assessed by western blotting (Fig. 1C). $\alpha$-SMA localizes to the cytoplasm in cancer-derived fibroblasts (Fig. 1B). Western blotting demonstrated increased expression levels of $\alpha$-SMA in two cancer-derived fibroblast samples compared with two NEF samples. Therefore, the cancer-derived fibroblasts isolated in this study possessed typical CAF characteristics, and were confirmed to be CAFs.

Suppression of NK cell-killing activity by CAFs. We assessed the effect of CAFs on the killing activity of NK cells to investigate the mechanism of CAF-mediated immune evasion. Assessment of NK cell activity is often performed by measuring allogenic NK cell killing activity against K562 cells (14). Using this NK cell-killing assay, we first examined whether CAFs isolated from endometrial cancer suppressed the killing activity of allogenic NK cells (Fig. 2). NK cells co-cultured with CAFs were assessed for killing activity by changing the E:T (NK cell: K562 cell) ratio. The proportion of 


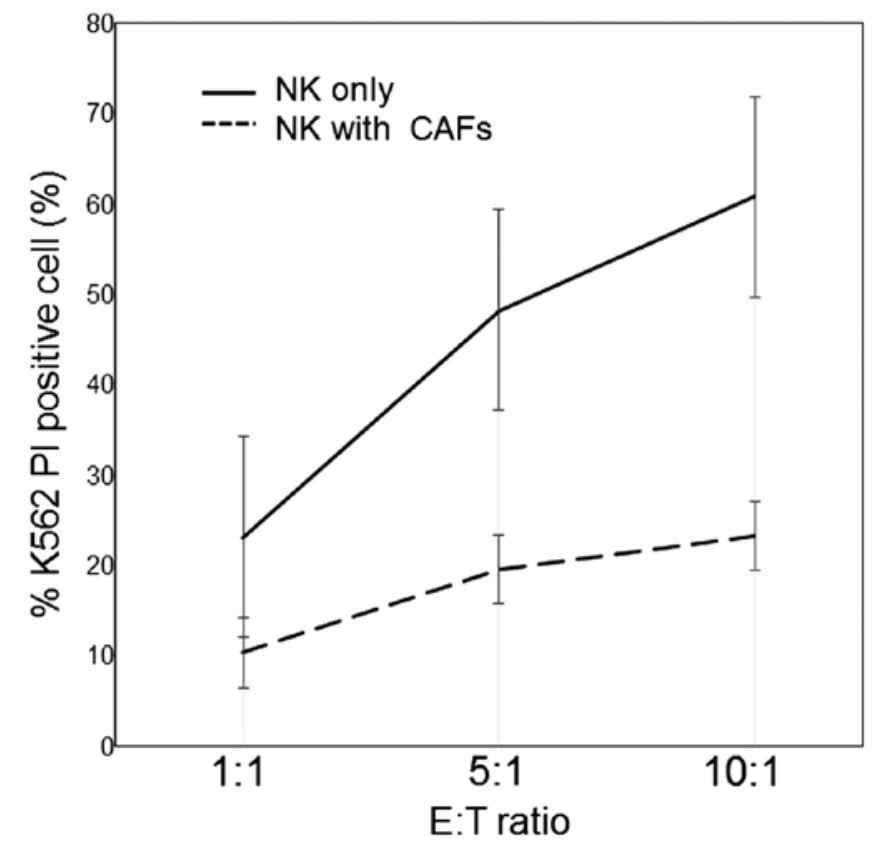

Figure 2. Suppression of NK cell killing activity by CAFs. NK cells cocultured with CAFs were assessed for killing assay changing E:T (NK cell: K562 cell) ratio. Percentage of dead K562 cell (K562 PI) was increased with increase of E:T ratio in either NK cell alone or NK cell co-cultured with CAFs. The killing activity of NK cells co-cultured with CAFs was significantly reduced, to less than one third the level of NK cells alone.

dead K562 cells increased in an E:T ratio-dependent manner in the presence of NK cells alone, or with NK cells co-cultured with CAFs, indicating that the target (K562) cells were killed by allogenic effector (NK) cells (Fig. 2). The killing activity of NK cells co-cultured with CAFs was significantly reduced, to less than one third the level of NK cells alone (Fig. 2). Next, NK cell activity was assessed when co-cultured with either NEFs or CAFs (Fig. 3). Allogenic NK cells were incubated for 24 hours with NEFs, CAFs, or no fibroblasts, and then exposed to K562 cells. NK cell-killing activity was determined by calculating the ratio of PI-positive K562 cells in the presence of NK cells co-cultured with fibroblasts versus NK cells that had been cultured alone. There was no significant difference in killing activity observed between NK cells only and NK cells co-cultured with NEFs. Again, the killing activity of NK cells co-cultured with CAFs was significantly decreased, to approximately one-third of the activity of NK cells cultured with no fibroblasts. However, NK cell activity was significantly decreased when co-cultured with CAFs, compared with NEF's (Fig. 3).

The CAF-induced decrease in NK cell activity was not rescued by indoleamine 2,3-dioxygenase inhibitor. Some studies have shown that malignant cells suppress NK cell activity via production of IDO $(15,16)$. IDO expression was barely detected in non-stimulated CAFs, whereas it was clearly detected in CAFs co-cultured with NK cells (data not shown). Previous reports have demonstrated that IDO expression in CAFs is likely caused by NK cell-derived IFN $\gamma$ (17). Therefore, we inhibited IDO activity using the IDO inhibitor 1-MT, to investigate whether IDO was involved in the suppression of NK

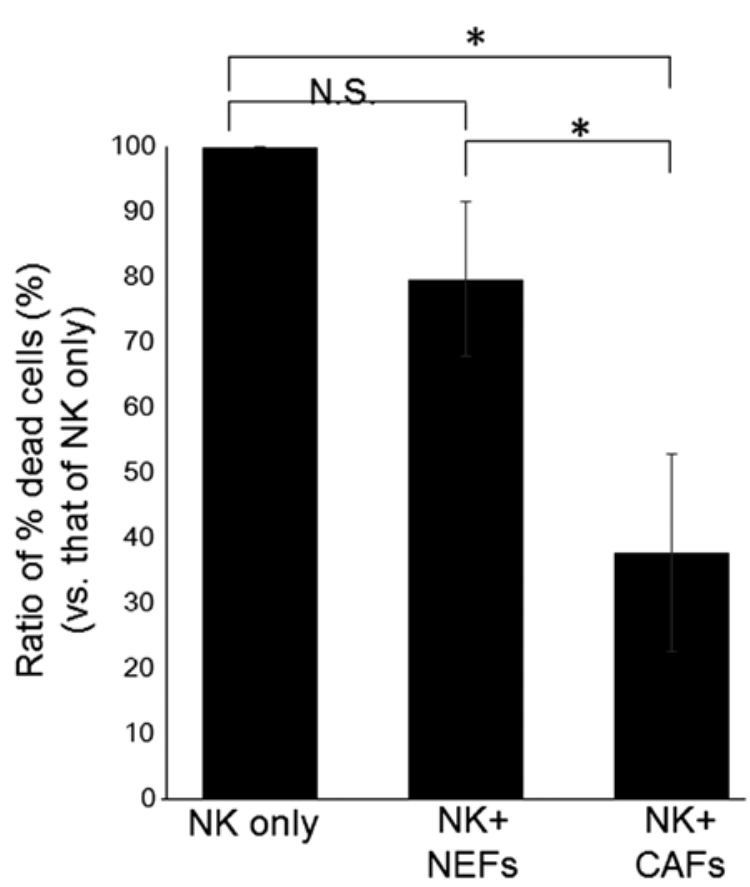

Figure 3. Comparison in NK cell activity between NK cell co-cultured with NEFs and CAFs. Comparison in NK cell activity between NK cell cocultured with NEFs and CAFs was made by the killing assay. The killing activity of NK cells with CAFs was significantly decreased with one third of that of NK cells only. The difference in the NK cell activity between NK cells with CAFs and NEFs was also significant. NS, not significant. Asterisk indicates p-value of $<0.05$.

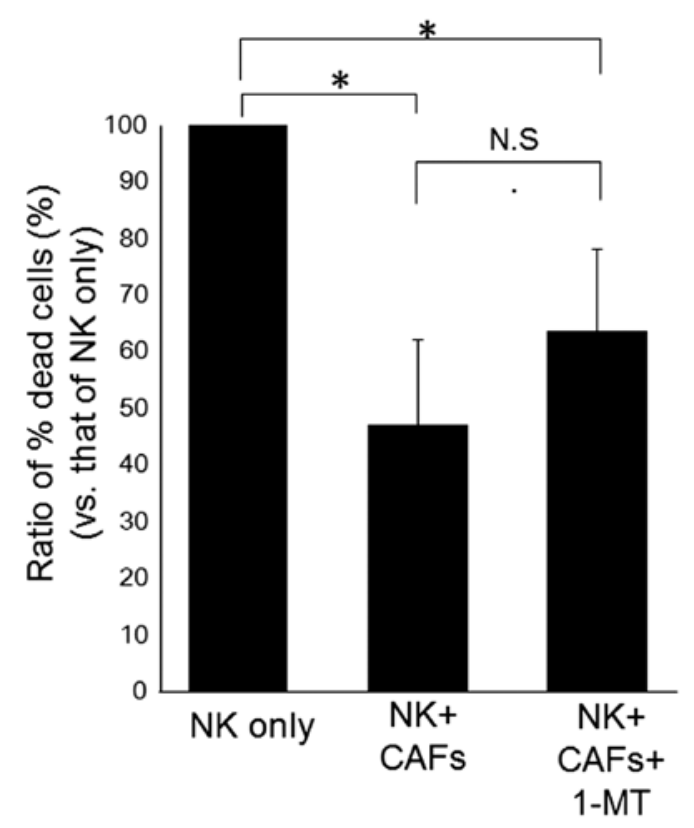

Figure 4. Decreased NK cell activity by CAFs was not rescued by indoleamine 2,3-dioxygenase inhibitor. IDO activity was blocked by 1-MT, an inhibitor of IDO, to examine whether IDO was involved in suppression of NK cell activity. The suppression of NK cell activity by CAFs was not rescued by 1 -MT treatment. NS, not significant. Asterisk indicates p-value of $<0.05$.

cell activity by CAFs. NK cells and CAFs were co-cultured in the presence or absence of 1-MT, followed by assessment of NK cell-killing activities (Fig. 4). While NK cell activity was 


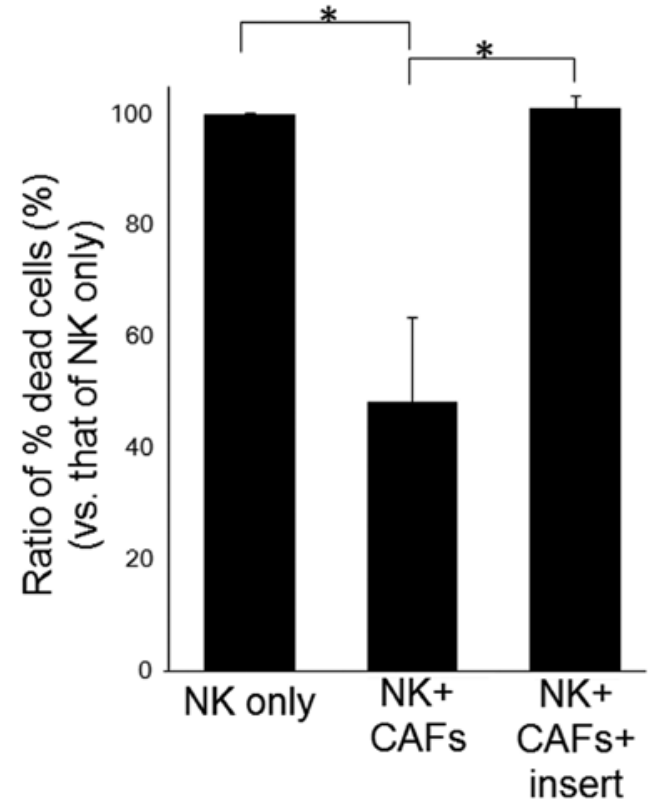

Figure 5. Cell-to-cell interaction was critical for the decreased NK cell activity by CAFs. NK cells and CAFs were cultured in a chamber with insert membrane to separate these cells. The suppression of NK cell activity by CAFs was completely canceled by blockage of cell-to-cell interaction using insert membrane. Asterisk indicates p-value of $<0.05$.

decreased by co-culture with CAFs, this suppression was not rescued by $1-\mathrm{MT}$ treatment.

Cell-to-cell interaction was critical for the CAF-induced decrease in NK cell activity. To examine whether direct cellto-cell interaction between NK cells and CAFs was required for the suppression of NK cell activity, NK cells and CAFs were cultured in a chamber with an inserted membrane sepa- rating these cells (Fig. 5). The suppression of NK cell activity by CAFs was completely abolished by the physical blockage of cell-to-cell interaction using the inserted membrane. These data strongly suggested that CAFs suppressed NK cell activity via cell-to-cell interaction rather than IDO, or other cytokine, production.

Cell-surface expression of PVR is decreased in CAFs compared with NEFs. We next focused on the cell-surface ligands expressed on CAFs that interact with activating NK cell receptors. Several ligands of paired or activating NK receptors have been previously demonstrated to be expressed on the cell surface of target cells, including malignant cells (18). However, few reports have investigated the cell-surface expression of $\mathrm{NK}$ receptor ligands on CAFs. We investigated the expression of several NK receptor ligands in CAFs. Due to the difference between CAFs and NEFs in their ability to induce NK cell activity, we hypothesized that the expression of NK receptor ligands may differ between CAFs and NEFs. Expression of poliovirus receptor (PVR/CD155), a ligand of paired NK receptors DNAM-1 and TIGIT, was observed to differ between CAFs and NEFs (Fig. 6). Flow cytometry analysis revealed that the cell-surface expression of PVR was decreased in CAFs compared with NEFs. This suggested that the downregulation of PVR in CAFs may mediate the suppression of DNAM-1 signaling.

PVR knockdown downregulates NK cell activity. To confirm whether PVR downregulation results in the decrease of NK cell-killing activity, PVR expression in NEFs was knocked down using siRNA against PVR (PVRsi) (Fig. 7A). PVRsi-transfected NEFs showed decreased cell-surface PVR expression when compared with control siRNA-transfected NEFs. The median flow cytometry index (MFI) of PVR expression in PVR knockdown cells was similar to that of

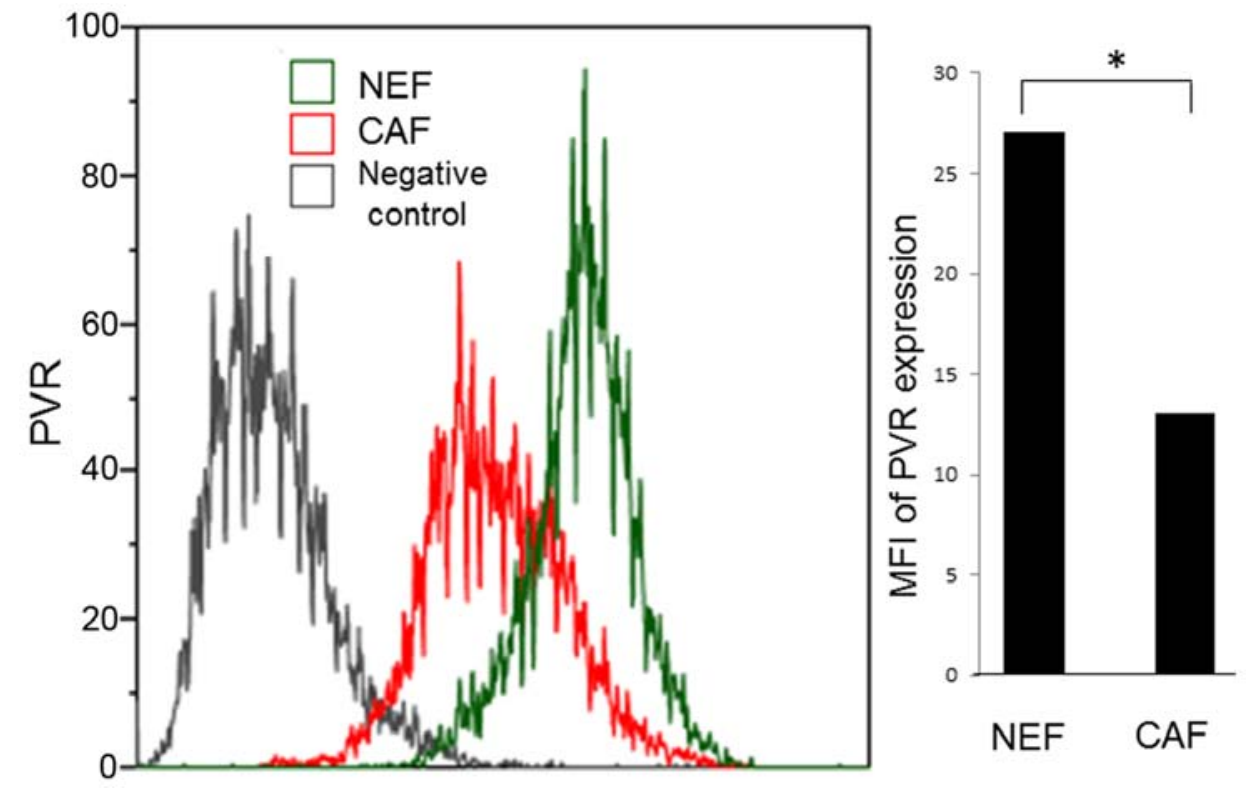

Figure 6. Cell-surface expression of PVR/CD155 in CAFs and NEFs. Expression of poliovirus receptor (PVR/CD155), a ligand of paired NK receptors (DNAM-1 and TIGIT), differed between CAFs and NEFs. Flow cytometry analysis revealed that PVR expression was clearly decreased in CAFs compared with NEFs. Asterisk indicates p-value of $<0.05$. 


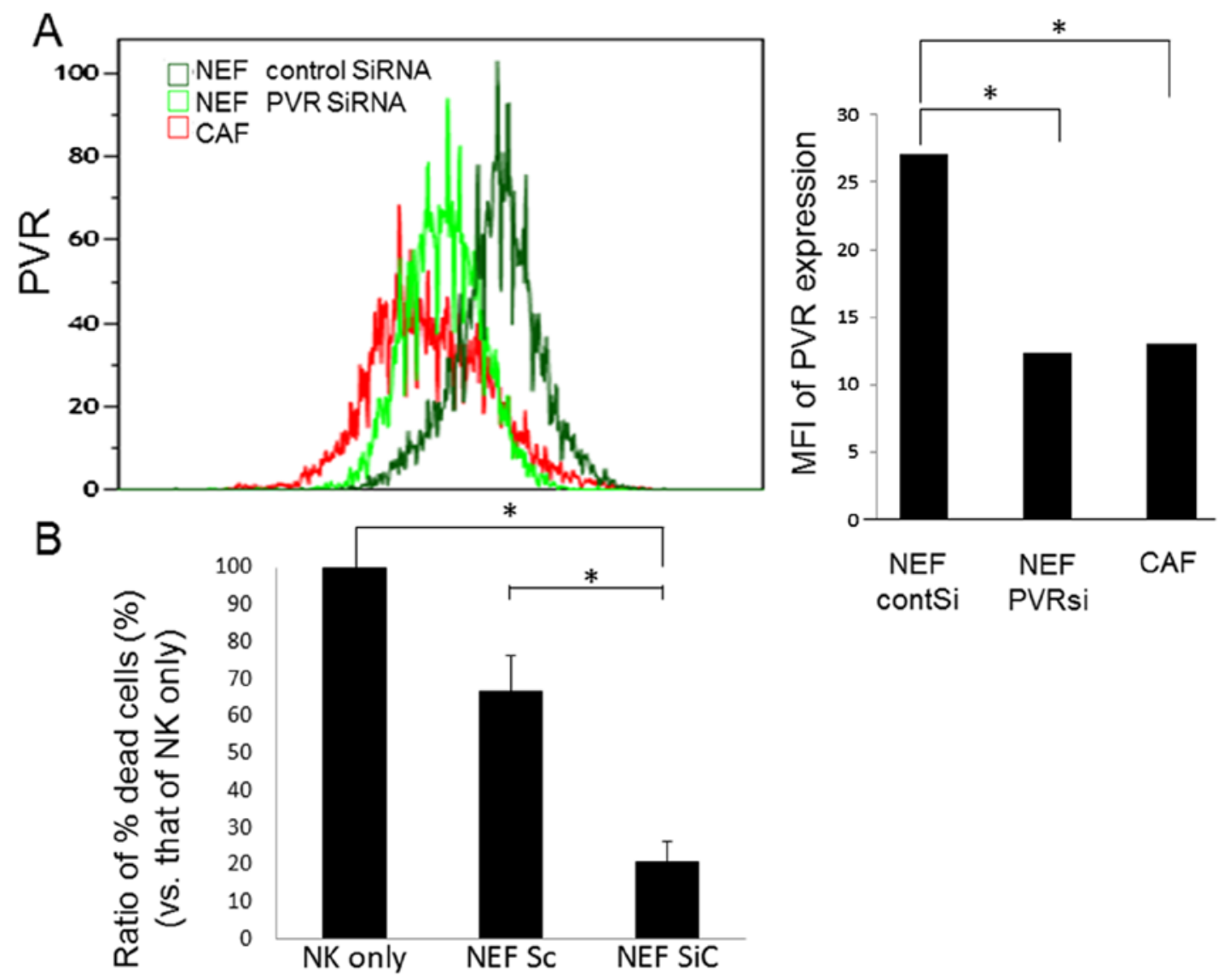

Figure 7. Knock-down of PVR/CD155 in NEFs provided the downregulation of NK cell activity. (A) PVR expression in NEFs was knocked down by transduction of siRNA for PVR (PVRsi) into NEFs. (B) NK cell killing activity of NK cells co-cultured with PVRsi-transduced NEFs was decreased to approximately one third of that with control si-transduced NEFs. Asterisk indicates p-value of $<0.05$.

CAFs (Fig. 7A). Interestingly, NK cell-killing activity of NK cells co-cultured with PVRsi-transfected NEFs was decreased to approximately one third of that of control si-transfected NEFs (Fig. 7B). The decreases in both PVR expression and effect on NK cell activity were approximately equivalent to those of CAFs. These data suggested that the level of PVR cell-surface expression in CAFs was critical for the killing activity of NK cells.

\section{Discussion}

We demonstrated that CAFs showed increased suppression of NK cell-killing activity compared with NEFs, due to decreased PVR cell surface expression, a ligand of an NK activating receptor. In this study, human CAFs and NEFs were isolated from the stroma of endometrial cancer and normal endometrium, respectively, and their interactions with NK cells were compared. Uterine endometrium is composed of many glands and abundant stroma. The endometrial stroma is enriched in fibroblasts, and surrounds the endometrial glandular epithelium. Endometrial cancer cells can transform normal fibroblasts in the stroma into CAFs. In the cancer microenvironment, CAFs acquire cancer-specific characteristics in addition to their fibroblastic background. Therefore, we believe that the comparison between endometrial CAFs and normal endometrial stromal fibroblasts was suitable for investigation of
CAF characteristics. We observed an increased $\alpha$-SMA expression level in CAFs compared with NEFs, in confirmation with previous studies demonstrating the expressing of $\alpha$-SMA and vimentin in active CAFs (19). CD90 is a fibroblastic marker that is also expressed in CAFs of a variety of cancers, such as lung, prostate, pancreas, and breast cancers (20-22). CAFs isolated from endometrial cancer in this study were fibroblasts showing typical CAF characteristics.

Many previous studies have demonstrated the suppression of NK cell activity by cancer cells by measuring the killing activity of allogenic NK cell against K562 cells (23). Some studies have shown that malignant cells suppress NK cell activity via the production of IDO. In this study, the suppression of NK cell-killing activity by CAFs was not rescued by exposure to 1-MT (IDO inhibitor), suggesting suppression of NK cell activity is not due to IDO production by CAFs. Prevention of cell-to-cell interaction between NK cells and CAFs completely abolished the suppression of NK cell activity by CAFs, indicating direct interaction between NK cells and CAFs was critical for the CAF-induced suppression of NK cell activity. Several studies have demonstrated that exosomes derived from CAFs may interact with NK cells to suppress NK activity. However, exosomes should be able to penetrate the transwell membrane used in this study, suggesting that the suppression of NK cell activity was not dependent on CAF exosome exposure. 
Our data demonstrated cell-surface expression of PVR was reduced in CAFs, compared with NEFs. The MFI of PVR in CAFs was half that in NEFs. NK activating receptors include NKp30, NKp44, NKp46, NKG2D, DNAM-1, and LFA-1 (8). Among the ligands of these receptors, PVR, a ligand of DNAM-1, was observed in this study to be ubiquitously expressed on the cell-surface of CAFs. It is known that DNAM-1 activates NK cell activity. We observed that co-culture of NK cells and CAFs did not alter DNAM-1 expression in NK cells (data not shown). In contrast, PVR interacts with NK paired receptors, DNAM-1 (activating) and TIGIT (inhibiting). Interestingly, some studies demonstrated that PVR interacts with higher affinity to TIGIT than DNAM-1. Our data suggested that the reduced expression of PVR in CAFs resulted in decreased interaction with DNAM-1, leading to suppression of NK killing activity. Furthermore, it is possible that the high affinity of PVR to TIGIT allowed even low expression of cell-surface PVR in CAFs to bind to TIGIT, thus inhibiting NK cell activity. We also demonstrated that the NK killing activity of PVR-knockdown NEFs decreased to levels comparable with that of CAFs. The data suggested that the reduction of PVR expression in CAFs is critical for CAF-induced suppression of NK cell activity.

Many studies have reported PVR involvement in NK cell-associated immune-evasion by malignant cells, and that low expression of PVR is associated with poor prognosis (24). However, this study is the first to demonstrate that the suppression of NK killing activity by CAFs was mediated by downregulation of cell-surface PVR in CAFs; however, the mechanism of PVR downregulation remains to be clarified. Recently, a study by Gong et al reported that ER stress induces the downregulation of PVR in hepatoma cells via the ATF6 and IREla pathways (25). ER stress, such as that induced by hypoxia in the TME, may result in downregulation of PVR in CAFs; therefore a low-level of PVR expression in fibroblasts may be a characteristic of CAFs. Further study is warranted to bring to light the mechanism by which CAFs acquire such features in the TME.

CAFs may assist malignant cells in a similar fashion to maintain the immunosuppressive microenvironment of the tumor. Additionally, immune evasion may be influenced by both CAFs and malignant cells with regard to NK cell-mediated killing activity. Therefore, soluble PVR may be used as a potential agent to activate NK cell activity in the TME. These data may provide a novel strategy for inhibiting the immune evasion system in the TME.

In conclusion, this is the first report to demonstrate that CAF-mediated suppression of NK killing activity is due to downregulation of PVR cell-surface expression in CAFs. We discovered that CAFs suppressed NK cells function via a receptor-ligand interaction, aiding cancer progression. Soluble PVR may be used as a potential agent to activate NK cell activity in the TME. These data may provide a novel strategy for inhibiting the immune evasion system in the TME.

\section{Acknowledgements}

The authors would like to thank Dr Terufumi Yokoyama for expert advice on experimental methodologies. This study was supported by a grant-in-aid from the Ministry of Health,
Labour and Welfare of Japan (KK) and by a cancer research grant from the Ministry of Education, Culture, Sports, Science and Technology (K.K., K.A. and K.T.) of Japan.

\section{References}

1. Karagiannis GS, Poutahidis T, Erdman SE, Kirsch R, Riddell RH and Diamandis EP: Cancer-associated fibroblasts drive the progression of metastasis through both paracrine and mechanical pressure on cancer tissue. Mol Cancer Res 10: 1403-1418, 2012.

2. Lorusso $\mathrm{G}$ and Rüegg C: The tumor microenvironment and its contribution to tumor evolution toward metastasis. Histochem Cell Biol 130: 1091-1103, 2008.

3. Joyce JA and Pollard JW: Microenvironmental regulation of metastasis. Nat Rev Cancer 9: 239-252, 2009.

4. Franco OE, Shaw AK, Strand DW and Hayward SW: Cancer associated fibroblasts in cancer pathogenesis. Semin Cell Dev Biol 21: 33-39, 2010.

5. Kalluri R and Zeisberg M: Fibroblasts in cancer. Nat Rev Cancer 6: 392-401, 2006.

6. Sanz-Moreno V, Gaggioli C, Yeo M, Albrengues J, Wallberg F, Viros A, Hooper S, Mitter R, Féral CC, Cook M, et al: ROCK and JAK1 signaling cooperate to control actomyosin contractility in tumor cells and stroma. Cancer Cell 20: 229-245, 2011.

7. Gaggioli C, Hooper S, Hidalgo-Carcedo C, Grosse R, Marshall JF, Harrington K and Sahai E: Fibroblast-led collective invasion of carcinoma cells with differing roles for RhoGTPases in leading and following cells. Nat Cell Biol 9: 1392-1400, 2007.

8. Chan CJ, Smyth MJ and Martinet L: Molecular mechanisms of natural killer cell activation in response to cellular stress. Cell Death Differ 21: 5-14, 2014.

9. Calon A, Tauriello DV and Batlle E: TGF-beta in CAF-mediated tumor growth and metastasis. Semin Cancer Biol 25: 15-22, 2014.

10. Salaroglio IC, Campia I, Kopecka J, Gazzano E, Orecchia S, Ghigo D and Riganti C: Zoledronic acid overcomes chemoresistance and immunosuppression of malignant mesothelioma. Oncotarget 6: 1128-1142, 2015.

11. Tahara-Hanaoka S, Shibuya K, Onoda Y, Zhang H, Yamazaki S, Miyamoto A, Honda S, Lanier LL and Shibuya A: Functional characterization of DNAM-1 (CD226) interaction with its ligands PVR (CD155) and nectin-2 (PRR-2/CD112). Int Immunol 16: $533-538,2004$

12. Bottino C, Castriconi R, Pende D, Rivera P, Nanni M, Carnemolla B, Cantoni C, Grassi J, Marcenaro S, Reymond N, et al: Identification of PVR (CD155) and Nectin-2 (CD112) as cell surface ligands for the human DNAM-1 (CD226) activating molecule. J Exp Med 198: 557-567, 2003.

13. Atsumi S, Matsumine A, Toyoda H, Niimi R, Iino T and Sudo A: Prognostic significance of CD155 mRNA expression in soft tissue sarcomas. Oncol Lett 5: 1771-1776, 2013.

14. Tanizaki Y, Kobayashi A, Toujima S, Shiro M, Mizoguchi M, Mabuchi Y, Yagi S, Minami S, Takikawa $\mathrm{O}$ and Ino $\mathrm{K}$ : Indoleamine 2,3-dioxygenase promotes peritoneal metastasis of ovarian cancer by inducing an immunosuppressive environment. Cancer Sci 105: 966-973, 2014.

15. Kasatori N, Ishikawa F, Ueyama M and Urayama T: A differential assay of NK-cell-mediated cytotoxicity in K562 cells revealing three sequential membrane impairment steps using three-color flow-cytometry. J Immunol Methods 307: 41-53, 2005.

16. Yoshida $\mathrm{N}$, Ino $\mathrm{K}$, Ishida $\mathrm{Y}$, Kajiyama $\mathrm{H}$, Yamamoto $\mathrm{E}$, Shibata K, Terauchi M, Nawa A, Akimoto H, Takikawa O, et al: Overexpression of indoleamine 2,3-dioxygenase in human endometrial carcinoma cells induces rapid tumor growth in a mouse xenograft model. Clin Cancer Res 14: 7251-7259, 2008.

17. Löb S, Königsrainer A, Rammensee HG, Opelz G and Terness P: Inhibitors of indoleamine-2,3-dioxygenase for cancer therapy: Can we see the wood for the trees? Nat Rev Cancer 9: 445-452, 2009.

18. Pegram HJ, Andrews DM, Smyth MJ, Darcy PK and Kershaw MH: Activating and inhibitory receptors of natural killer cells. Immunol Cell Biol 89: 216-224, 2011.

19. Augsten M: Cancer-associated fibroblasts as another polarized cell type of the tumor microenvironment. Front Oncol 4: 62, 2014.

20. Yan X, Luo H, Zhou X, Zhu B, Wang Y and Bian X: Identification of CD90 as a marker for lung cancer stem cells in A549 and H446 cell lines. Oncol Rep 30: 2733-2740, 2013. 
21. True LD, Zhang H, Ye M, Huang CY, Nelson PS, von Haller PD, Tjoelker LW, Kim JS, Qian WJ, Smith RD, et al: CD90/THY1 is overexpressed in prostate cancer-associated fibroblasts and could serve as a cancer biomarker. Mod Pathol 23: 1346-1356, 2010.

22. Lu H, Clauser KR, Tam WL, Fröse J, Ye X, Eaton EN, Reinhardt F, Donnenberg VS, Bhargava R, Carr SA, et al: A breast cancer stem cell niche supported by juxtacrine signalling from monocytes and macrophages. Nat Cell Biol 16: 1105-1117, 2014.

23. Alter G, Malenfant JM and Altfeld M: CD107a as a functional marker for the identification of natural killer cell activity. $\mathrm{J}$ Immunol Methods 294: 15-22, 2004.
24. Qu P, Huang X, Zhou X, Lü Z, Liu F, Shi Z, Lü L, Wu Y and Chen Y: Loss of CD155 expression predicts poor prognosis in hepatocellular carcinoma. Histopathology 66: 706-714, 2015.

25. Gong J, Fang L, Liu R, Wang Y, Xing J, Chen Y, Zhuang R, Zhang Y, Zhang C, Yang A, et al: UPR decreases CD226 ligand CD155 expression and sensitivity to NK cell-mediated cytotoxicity in hepatoma cells. Eur J Immunol 44: 3758-3767, 2014. 